\title{
Role of CSE1034 in Escherichia coli Biofilm Destruction
}

\author{
Manu Chaudhary, Shailesh Kumar and Anurag Payasi* \\ Department of Cell Culture and Molecular Biology, Venus Medicine Research Centre, Baddi, HP, India
}

\begin{abstract}
Present study was conducted to assess the effect of ethylenediamine tetraacetic acid (EDTA); a non antibiotic adjuvant, and CSE1034, a novel antibiotic adjuvant entity in biofilm destruction of Escherichia coli, and comparing the efficacy with other drugs. We first determined the susceptibility of six antimicrobial agents against planktonic cultures, as well as sessile cells of E. coli clinical isolates, using the Clinical and Laboratory Standards Institute (CLSI) method. Subsequently, effects of EDTA alone, and drugs on bacterial curli production, adhesion, and in-vitro biofilm destruction were studied. The percentage of biofilm persistence was determined with spectrophotometry. The structural damage of biofilms was studied by scanning electron microscope. It was found that, among the drugs used, CSE1034 was the most effective against all of the $E$. coli clinical isolates, with MIC and MBEC values ranging from $32-64 \mu \mathrm{g} / \mathrm{ml}$ and $256-512 \mu \mathrm{g} / \mathrm{ml}$, respectively. Exposure of clinical isolates with EDTA alone caused inhibitions of curli formation and bacterial adhesion at 4 to $5 \mathrm{mM}$. Further, EDTA treatment of preformed biofilm caused complete biofilm destruction at 8-10 mM. Interestingly, CSE1034 due to presence of $10 \mathrm{mM}$ EDTA led to enhanced antibacterial, as well as biofilm destruction activities. Results of spectrophotometric analysis and scanning electron microscopy revealed that approximately $92 \%$ biofilms were eradicated by the CSE 1034 , and not by comparator drugs. The results indicate that the CSE1034 appears to be most effective drug of choice for the treatment of infections caused by $E$. coli.
\end{abstract}

Keywords: Adjuvant; Biofilm; Curli; CSE1034

\section{Introduction}

Enterobacteriaceae is a large family of Gram-negative bacteria, that includes many harmless symbiont bacteria and pathogenic, such as Salmonella, Escherichia coli, Yersinia pestis, Klebsiella and Shigella. E. coli is known to be a leading species amongst facultative anaerobic bacteria, and is main causative agents of cholecystitis, bacterimia, urinary tract infections (UTIs), meningitis and pneumonia. It has been reported that many isolates of E. coli produce biofilm structures in vivo and in vitro [1], and approximately $90 \%$ of UTIs are caused by E. coli biofilms [2]. It has been reported that curli, a very thin, coiled extracellular appendages expressed on the surface of most E. coli and Salmonella enteria strains which bind fibronectin and other proteins, are required for the biofilm development $[3,4]$.

The adhesion of the bacterial cell to any surface is an initial step of biofilm formation $[5,6]$. It is thought that the curli causes cell to surface interactions, leading to adherence of bacterial cells to any of the solid surface, including living tissues, in dwelling medical devices, dental water unit systems $[1,4,5]$, and turning the planktonic growth to a sessile biofilm mode of growth $[1,6,7]$. Once the biofilm is developed, the sessile bacterial cells start to exhibit more resistance to antibacterial agents than that of planktonic cells [2,8], leading to failure of antibacterial therapy. Low penetration of antibacterial agents through the polymer matrix of the biofilm, is one of the important factors contributing to antibiotic resistance in E. coli $[4,9,10]$. However, many antibacterial agents have been used to eradicate the E. coli biofilm $[11,12]$, none so far has been demonstrated to eradicate biofilm effectively. The growing rate of resistance of antibacterial agents to $E$. coli biofilm led us to conduct a study on a new antibiotic adjuvant entity (AAE), which could be effective against $E$. coli biofilm. We studied a non-antibiotic adjuvant, ethylenediamine tetraacetic acid disodium (EDTA), alone along with ceftriaxone and sulbactam, which combinely was termed as CSE1034.

Therefore, the main objective of the present study was to evaluate the effect of EDTA and CSE1034 in curli production, adhesion and biofilm destruction of E. coli, and a comparison with other antibacterial drugs. For this purpose, we used seventeen extended spectrum $\beta$-lactamases (ESBL) producing clinical isolates, and six antibacterial agents: CSE1034, ceftriaxone, amoxycillin plus clavulanic acid, meropenem, cefoperazone+sulbactam and piperacillin+tazobactam. In addition, biofilm destruction with and without treatment of drugs were observed by scanning electron microscopy (SEM).

\section{Materials and Methods}

\section{Bacterial strains}

Seventeen clinical isolates collected from patients with UTIs, bacteremia and pneumonia used in this study were obtained from Vijayanagara Institute of Medical Sciences, Bareilly, U.P, India, Postgraduate Institute of Medical Science (PGIMS), Lucknow, India, and Government Medical College and Hospital, Chandigarh, India. The identity of clinical isolates was re-confirmed by using VITEK-2. Prior to use, bacterial suspensions were spread onto Mueller-Hinton solid medium (MHSM; Himedia, Mumbai, India), and incubated at $35^{\circ} \mathrm{C}$ for $24 \mathrm{hrs}$. The ESBL confirmation was also done using Clinical and Laboratory Standards Institute (CLSI) methods [13]. The bacterial suspension, at a concentration of $10^{6}$ colony-forming units $(\mathrm{cfu} / \mathrm{ml})$, were used for susceptibility studies, adhesion study, curli formation and biofilm formation.

*Corresponding author: Anurag Payasi, Department of Cell Culture and Molecular Biology, Venus Medicine Research Centre, Hill Top Industrial Estate, Bhatoli Kalan, Baddi, HP-173205, India, Tel: 91-1795-302005; Fax: 91-1795302133; E-mail: ccmb@venusremedies.com

Received March 12, 2013; Accepted April 17, 2013; Published April 22, 2013

Citation: Chaudhary M, Kumar S, Payasi A (2013) Role of CSE1034 in Escherichia coli Biofilm Destruction. J Microb Biochem Technol 5: 054-058. doi:10.4172/19485948.1000100

Copyright: (c) 2013 Chaudhary M, et al. This is an open-access article distributed under the terms of the Creative Commons Attribution License, which permits unrestricted use, distribution, and reproduction in any medium, provided the original author and source are credited 


\section{Antibacterial agents}

A novel antibiotic adjuvant entity (AAE), with ceftriaxone sodium plus $10 \mathrm{mM}$ EDTA disodium plus sulbactam sodium herein, after referred to as CSE1034 (Elores; Venus Remedies Limited, Chandigarh, India), ceftriaxone (Rocephion, Hoffmann-Laroche Pharmaceutiical Limited, Basel Switzerland), amoxycillin plus clavulanic acid (Augmentin, Glaxo Smith Kline, Pharmaceuticals Limited, Mumbai, India), piperacilline plus tazobactam (Zosyn; Wyeth Pharmaceuticals, Mumbai, India), meropenem (Meronem, Astrazeneca Pharma India Ltd., Banglore, India) cefoperazone plus sulbactam (Magnex, Pfizer Limited, Mumbai, India), were used in the study. EDTA disodium was procured from Himedia, Mumbai, India. All the drugs were reconstituted according to instructions of manufacturer, prior to use. Working solutions were prepared using MH broth (Mueller Hinton, Himedia, Mumbai, India), and serial two fold dilutions were made using CAMH (Cation-Adjusted Mueller-Hinton, Himedia, Bombay, India) broth in wells of 96-well plate.

\section{Antimicrobial susceptibility}

MIC: Minimum inhibitory concentration (MIC) was performed according to CLSI [13]. E. coli MTCC 739 was used as a control.

AST: The following antibiotics were used in this study: ceftriaxone plus disodium edetate plus sulbactam; CSE1034 (Elores) (30:10:15 $\mu \mathrm{g})$, ceftriaxone $(30 \mu \mathrm{g})$, cefoperazone+sulbactam $(75: 30 \mu \mathrm{g})$, piperacillin plus tazobactam (100:10 $\mu \mathrm{g})$, amoxycillin plus clavulanic acid (20:10 $\mu \mathrm{g})$, and meropenem $(10 \mu \mathrm{g})$. All the discs were obtained from $\mathrm{Hi}$ Media Laboratories Pvt. Ltd., Mumbai, India.

\section{Effect of EDTA and drugs on curli production assay}

Curli formation was studied in all isolates using a congo red agar (CRA) plate, as described previously [14]. To study the effect of EDTA disodium and drugs on curli production, 1.25 to $20 \mathrm{mM}$ EDTA, and half of MIC of drugs were used. Curli producing bacteria appeared as red colonies, whereas non-curli producers appeared as pink. E. coli O157:H7 deficient in production of curli (ATCC 43894-) was used as negative control, and its corresponding curli-producing strains $(43894+)$ was used as a positive control.

\section{Effect of EDTA and drugs on adhesion assay}

Adhesion study was done as described earlier using the same concentration of EDTA and drugs, as used in curli study. The minimal concentration of EDTA and drugs which inhibited adhesion process was determined visually as the concentration where the stained purple color was not observed. E. coli O157:H7 deficient in production of curli (ATCC 43894-) was used as negative control, and its corresponding curli-producing strains (43894+) was used as a positive control.

\section{Effect of EDTA and drugs on biofilm}

Biofilms of curli positive isolates were developed using Calgary Biofilm Device (CBD) [15]. Minimumbiofilm destruction concentration (MBEC) was determined, as reported elsewhere [16]. To assess the effects of EDTA and drugs on biofilms, the EDTA concentration was the same as mentioned above, but the concentrations of drugs were four times of their MIC.

\section{Quantification of biofilms}

The quantification of biofilms were done using the methods described earlier [16].

\section{Scanning electron microscopy}

The samples for SEM analysis were prepared as described earlier [4]. SEM images were captured using scanning electron microscope (Hitachi S3700-N). SEM images of antibiotic-exposed biofilm samples taken at a 10,000 magnification. SEM analysis was performed only on the selected groups treated with CSE1034, meropenem and piperacilline plus tazobactam.

\section{Reproducibility of the results}

Results presented here are average of three experiments (mean \pm SD), except SEM. Statistical analysis was performed with one-way analysis of variance (ANOVA) and Dunnett's test. Values of $\mathrm{P}>0.05$ were considered not to be significant.

\section{Results}

\section{ESBL identifications and MIC}

Identity confirmation and ESBL characterization results revealed that all of the isolates were of E. coli with ESBL positive. Among the drugs used, CSE1034 was most effective against all of the clinical isolates, with a MIC and MBEC values of about 32-64 and 256-512 $\mu \mathrm{g} /$ $\mathrm{ml}$, respectively. The second drug, meropenem, was effective to some extent, with a MIC and MBEC values of about 32-128 and 256-2048 $\mu \mathrm{g} / \mathrm{ml}$. Cefoperazone plus sulbactam and piperacillin plus tazobactam exhibited MIC values approximately $256-512$ and $128-1024 \mu \mathrm{g} / \mathrm{ml}$ respectively. The MBEC values for each of cefoperazone plus sulbactam and piperacillin plus tazobactam were $2048-4096 \mu \mathrm{g} / \mathrm{ml}$. However, the other drugs (ceftriaxone and amoxycillin plus clavulanic acid) were much more resistant, with a MIC and MBEC values $>512$ and $>4096$, respectively (Table 1).

AST: Results of AST study of various antimicrobial agents in curli positive isolates are shown in table 2. Among the drugs used in the study, CSE1034 was the only drug found to be most active, with $92.3 \%$ susceptibility against curli positive clinical isolates. Second most active antibacterial agent was meropenem with $61.5 \%$ susceptibility, followed by piperacillin plus tazobactam (46.1\%), ceftriaxone plus sulbactam (38.4\%), and each of ceftriaxone and amoxycillin plus clavulanic acid (30.7\%) (Table 2).

\section{Effect of EDTA and drugs on curli formation}

The isolates produced bright red colonies on CRA plate confirmed to be a curli producer, while isolate showed mild pink colonies considered to be non-curli producers. Among the seventeen clinical isolates, only thirteen (76\%) clinical isolates were curli producers.

\begin{tabular}{|l|l|l|}
\hline Name of Drugs/adjuvant & MIC $(\mu \mathrm{g} / \mathrm{ml})$ & $\begin{array}{l}\text { MBEC } \\
(\boldsymbol{\mu g} / \mathrm{ml})\end{array}$ \\
\hline CSE1034 & $32-64$ & $256-512$ \\
\hline Ceftriaxone & $>512$ & $>4096$ \\
\hline Cefoperazone+sulbactam & $256-512$ & $2048-4096$ \\
\hline Meropenem & $32-128$ & $256-2048$ \\
\hline Piperacillin plus tazobactam & $128-1024$ & $2048-4096$ \\
\hline Amoxycillin plus clavulanic acid & $>512$ & $>4096$ \\
\hline
\end{tabular}

$\mathrm{MIC}=$ Minimum Inhibitory Concentration; MBEC=Minimum Biofilm Eradication Concentration. MIC study of antibacterial agents was performed on all of the clinical isolates. MBEC study of antibacterial agents was performed on only thirteen clinical isolates which were positive for curli.

Table 1: MICs and MBECs of antibacterial agents for E. coli clinical isolates. 
Moreover, EDTA caused a concentration dependent inhibition in curli forming ability of clinical isolates, with a complete inhibition at 4-5 mM. Among the clinical isolates investigated, 10 clinical isolates showed a complete inhibition of curli formation at $5.0 \mathrm{mM}$, while remaining exhibited at $4.0 \mathrm{mM}$ of EDTA disodium. In positive control, E.coli O157:H7(ATCC43894+), curli formation was inhibited at 4.0 $\mathrm{mM}$. When various drugs were examined on the curli formation, interestingly, only CSE1034 could inhibit curli production.

\section{Effect of EDTA and drugs on adhesion}

Exposure of clinical isolates to EDTA resulted in a concentration dependent decrease in the bacterial cell adhesion, with complete inhibition at 4.0 to $5.0 \mathrm{mM}$ EDTA. Among the clinical isolates investigated, 10 clinical isolates showed a complete inhibition of adhesion at $5.0 \mathrm{mM}$, while remaining three exhibited at $4.0 \mathrm{mM}$ of EDTA disodium. The decreased in adhesion after treatment with EDTA was statistically significant $(\mathrm{p}<0.01)$, in comparison to control at concentration $2.5 \mathrm{mM}$ and above (Figure 1). In positive control, E.coli O157:H7 (ATCC43894+), bacterial adhesion was inhibited at 4.0 $\mathrm{mM}$. Similarly, when various drugs using half of MIC were studied on adhesion, only CSE1034 was inhibited bacterial adhesion, which was statistically significant.

\section{Effect of EDTA and drugs biofilm}

Matured biofilms of all E. coli clinical isolates were treated with different concentrations of EDTA disodium, and it was found that biofilm destruction was enhanced significantly $(\mathrm{p}<0.01)$, with increasing the concentration of EDTA disodium, and complete destruction was observed at 8.0 to $10 \mathrm{mM}$ EDTA disodium when used alone (Figure 2). Matured biofilms of $E$. coli strains treated with different drugs at 4 times of MIC, approximately to $92 \%$ of the biofilm was destroyed by the CSE1034 $(\mathrm{p}<0.01)$. The results demonstrated that the CSE1034 is much effective against $E$. coli biofilm than the other comparative drugs (Figure 3). Further, SEM analysis exhibited that CSE1034 treated group demonstrated ruptured biofilm and swollen cells (Figure 4).

\begin{tabular}{|l|l|l|l|}
\hline \multirow{2}{*}{ Antimicrobial agent } & \multicolumn{3}{l|}{ Percentage } \\
\cline { 2 - 4 } & Susceptible & Intermediate & Resistant \\
\hline CSE1034 & 92.3 & 7.7 & - \\
\hline Ceftriaxone & 30.7 & 15.4 & 53.8 \\
\hline Cefoperazone+sulbactam & 38.4 & 23.0 & 38.4 \\
\hline Meropenem & 61.5 & 23.0 & 15.4 \\
\hline Piperacillin plus tazobactam & 46.1 & 23.0 & 30.7 \\
\hline Amoxycillin plus clavulanic acid & 30.7 & 23.0 & 46.3 \\
\hline
\end{tabular}

Table 2: AST of the curli positive E. coli clinical isolates.

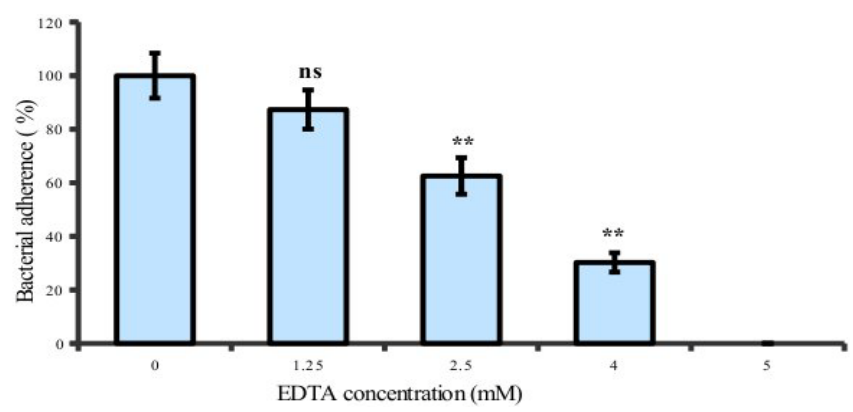

Figure 1: Effect of EDTA on adhesion of E. coli clinical isolates. Adherence inhibition significance levels- ns-p $>0.05$; ${ }^{*}-p<0.05$; ${ }^{* *}-p<0.01$, compared to control. The clinical isolates showing complete inhibition of bacterial adherence at 5 mM EDTA.

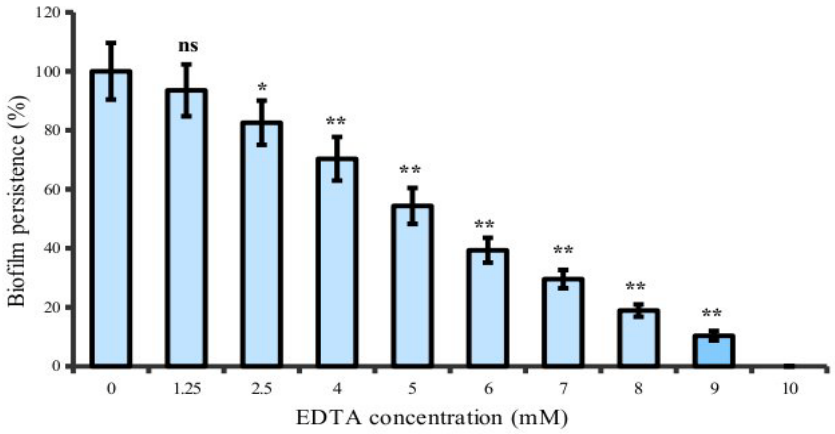

Figure 2: Percentage of $E$. coli clinical isolates biofilm persistence after exposure to EDTA. Biofilm eradication significance levels-ns-p $>0.05 ;{ }^{*}-p<0.05 ;{ }^{* *}-p<0.01$, compared to control. The clinical isolates showing complete biofilm eradication at $10 \mathrm{mM}$ EDTA.

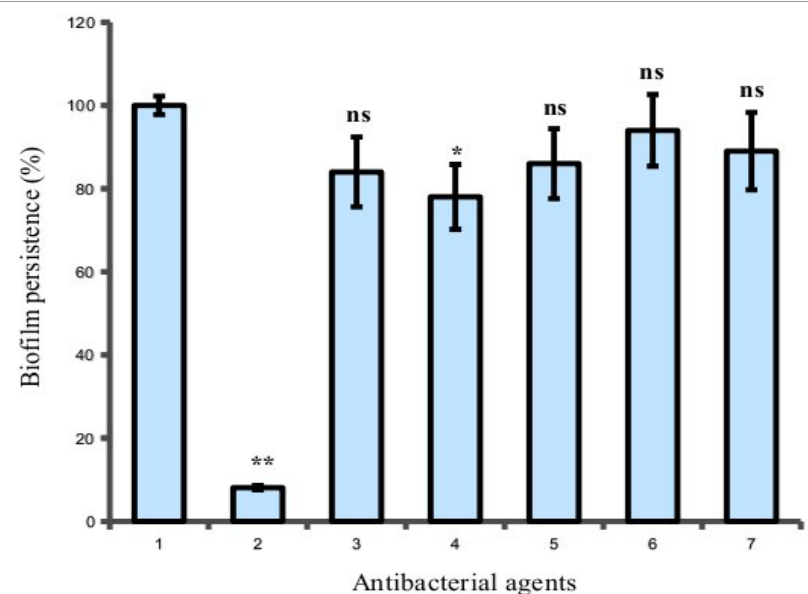

Figure 3: Percentage of $E$. coli clinical isolates biofilm persistence percentage in the presence of drugs. 1, control without antibiotic treatment, 2, CSE1034, 3, cefoperazone+sulbactam, 4, meropenem, 5, piperacillin plus tazobactam, 6 , ceftriaxone, 7 , Amoxycillin plus clavulanic acid. Biofilm persistence significance levels- ns-p $>0.05 ;{ }^{*}-p<0.05 ;{ }^{* *}-p<0.01$, compared to control.
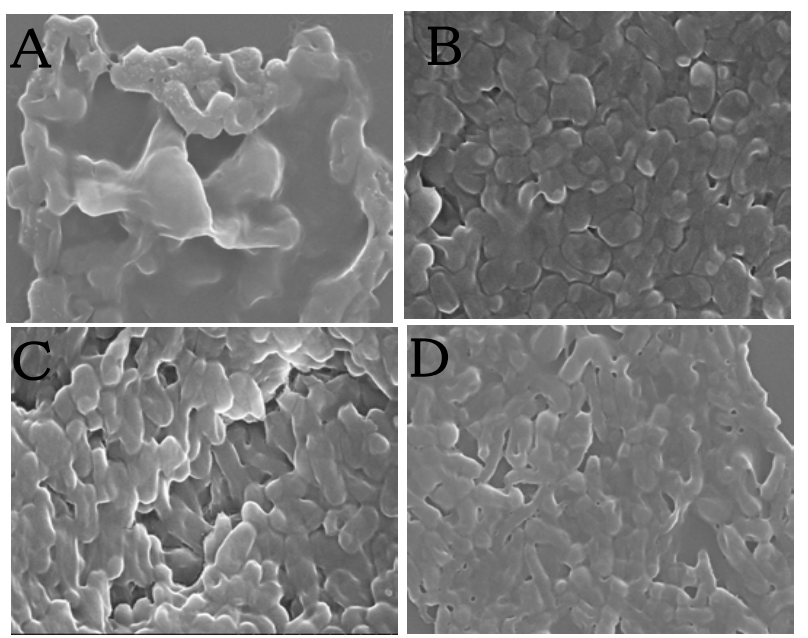

Figure 4: Scanning electron microscopy of one selected clinical isolates of $E$. colt biofilm on the surface of pegs of the microplate lid. (A) E. coli biofilm exposed to CSE1034 (significant disorganization of biofilm; a ruptured, porous and swollen cells; (B) E. coli biofilm exposed to piperacillin+tazobactam; (C) E.coli biofilm exposed to meropenem (little swollen cells in biofilm appeared); (D) control (without treatment). 


\section{Discussion}

E. coli is causing many serious complications in humans. Results obtained in the present investigation indicated the MBECs values of the drugs are several folds higher than their MICs, suggesting behavioral changes of bacterial cells towards antibiotic after biofilm formation. The results of our study demonstrated the least MICs and MBECs values of CSE1034, because of the synergistic activity of ceftriaxone plus sulbactam plus EDTA; the EDTA enhancing the penetration of drug by increasing the porosity, which in turn decreased in MICs, and further, EDTA also chelates divalent ions present in lipopolysaccharide layer of biofilms, thereby reducing MBECs value. However, other comparative drugs demonstrated very high MICs and MBECs values due to absence of EDTA.

It is well known that during biofilm formation, E. coli bacterial cells colonize on the surface through specific adhesin and curli $[1,14,17]$. Several authors have reported that curli promoting adhesion of the bacteria cells to the surface $[18,19]$, and adhesion is the first step for biofilm development [20]. However, our results revealed that 4-5 mM EDTA alone inhibited curli formation and bacterial adhesion. Similarly, among the tested drugs, only CSE1034 prevented the curli production as well as bacterial adhesion, due to presence of EDTA in CSE1034, whereas other comparative drugs including cefoperazone plus sulbactam, meropenem, piperacillin plus tazobactam, ceftriaxone and amoxycillin plus clavulanic acid failed to prevent curli formation and bacterial adhesion, indicating that the biofilm formation can't be controlled without inhibiting curli production and adhesion.

Earlier studies have shown that $50 \mathrm{mM}$ EDTA disrupts the biofilm [21-23]. Contrary to these, we found that $10 \mathrm{mM}$ EDTA disrupts the bacterial biofilm when tested alone; the concentration of EDTA used in our study was five times lower than previous study. There are many reports that the bacterial cells are covered by exopolysaccharide matrix, and this matrix is stabilized by divalent cations $[6,24,25]$.

The reduced efficacy of meropenem, piperacillin plus tazobactam, ceftriaxone, cefoperazone plus sulbactam and amoxycillin plus clavulanic acid towards E. coli biofilms, are due to presence of extracellular polysaccharides, to which these drugs could not penetrate. Previously, it has been reported that extracellular saccharides may prevent the diffusion of antibiotics across the biofilm [6]. However, CSE1034 destroyed approximately $92 \%$ E. coli biofilm, because EDTA may acts as a facilitating agent and helps penetration of antibiotic to biofilms formed by E. coli. This in turn leads to maximum destruction of the pre-formed biofilm exposed to CSE1034. EDTA removes divalent ions (e.g. $\mathrm{Mg}^{2+}$ and $\mathrm{Ca}^{2+}$ ), by chelating to cations of EPS and outer membrane of biofilms, and making the bacteria more susceptible to antibiotic. EDTA also causes loss of lipid, lipo-protein, fatty acids and polysaccharide from the biofilm, as well as bacterial cell wall. To our knowledge, the role of $E$. coli biofilm matrix in retarding the penetration of drugs has not been greatly investigated.

\section{Conclusion}

Results obtained in the present study revealed that low concentrations of EDTA (4-5 mM) prevent curli production and adhesion when used alone, and disrupts the pre-formed biofilm at almost double the concentration $(8-10 \mathrm{mM})$. Out of the various drugs tested, CSE1034 is the only drug which can eradicate the biofilms efficiently, and thus, it can be considered as a drug of choice in biofilm infections, including urinary tract infection, pneumonia and bacteremia caused by E. coli. The enhanced efficacy of CSE1034 in the eradication of biofilm infection is due to presence of EDTA, which helps in the destabilizing of the barriers responsible for the development of biofilm, as well as antibiotic resistance.

\section{Acknowledgement}

Authors are thankful to Venus Medicine Research Center, Germany AM Bahnhof 1-3, D-59368, Werne, Germany, for providing financial assistance to carry out the study. Authors are also thankful to Institute for providing clinical isolates.

\section{References}

1. Beloin C, Roux A, Ghigo JM (2008) Escherichia coli biofilms. Curr Top Microbio Immunol 322: 249-289.

2. Spurbeck RR, Stapleton AE, Johnson JR, Walk ST, Hooton TM, et al. (2011) Fimbrial profiles predict virulence of uropathogenic Escherichia coli strains: contribution of ygi and yad fimbriae. Infect Immun 79: 4753-4763.

3. May T, Tsuruta K, Okabe S (2011) Exposure of conjugative plasmid carrying Escherichia coli biofilms to male-specific bacteriophages. ISME J 5: 771-775.

4. Cegelski L, Pinkner JS, Hammer ND, Cusumano CK, Hung CS, et al. (2009) Small-molecule inhibitors target Escherichia coli amyloid biogenesis and biofilm formation. Nat Chem Biol 5: 913-919.

5. Macarisin D, Patel J, Bauchan G, Giron JA, Sharma VK (2012) Role of curli and cellulose expression in adherence of Escherichia coli $\mathrm{O} 157: \mathrm{H} 7$ to spinach leaves. Foodborne Pathog Dis 9: 160-167.

6. Boles BR, Horswill AR (2012) Swimming cells promote a dynamic environment within biofilms. Proc Natl Acad Sci U S A 109: 12848-12849.

7. Singh N, Agrawal V, Pemmaraju SC, Panwar R, Pruthi V (2011) Impact of infections Candida albicans biofilm on biomaterials. Indian J Biotechnol 10: 417-422.

8. Mahmoud HA, Melake NA, El-Semary MT (2012) Bactericidal Activity of various antibiotics versus tetracycline-loaded chitosan microspheres against Pseudomonas aeruginosa biofilms. Pharmaceut Anal Acta S15.

9. Donlan RM (2011) Biofilm elimination on intravascular catheters: importan considerations for the infectious disease practitioner. Clin Infect Dis 52: 10381045.

10. Bordi C, de Bentzmann S (2011) Hacking into bacterial biofilms: a new therapeutic challenge. Ann Intensive Care 1: 19.

11. Ponnusamy P, Natarajan V, Sevanan M (2012) In vitro biofilm formation by uropathogenic Escherichia coli and their antimicrobial susceptibility pattern. Asian Pac J Trop Med 5: 210-213.

12. Murugan S, Uma Devi P, Peedikayil NJ (2011) Antimicrobial susceptibility pattern of biofilm producing Escherichia coli of urinary tract infections. Curr Res Bacteriol 4: 73-80.

13. Clinical and Laboratory Standards Institute (CLSI) (2009) Performance standards for antimicrobial susceptibility testing. $19^{\text {th }}$ Informational Supplement, CLSI document M100-S19, CLSI 29, Wayne, PA, USA.

14. Reisner A, Haagensen JA, Schembri MA, Zechner EL, Molin S (2003) Development and maturation of Escherichia coli K-12 biofilms. Mol Microbio 48: 933-946.

15. Ceri H, Olson ME, Stremick C, Read RR, Morck D, et al. (1999) The calgary biofilm device: new technology for rapid determination of antibiotic susceptibilities of bacterial biofilms. J Clin Microbiol 37: 1771-1776.

16. Chaudhary M, Payasi A (2012) Alternative approach to increase antibiotic sensitivity of coagulase +ve and -ve Staphylococcus spp in planktonic and sessile cells. J Phar Res 5: 316-320.

17. Brombacher E, Baratto A, Dorel C, Landini P (2006) Gene expression regulation by the Curli activator CsgD protein: modulation of cellulose biosynthesis and control of negative determinants for microbial adhesion. J Bacteriol 188: 2027 2037.

18. Conceição RA, Ludovico MS, Andrade CG, Yano T (2012) Human sepsisassociated Escherichia coli (SEPEC) is able to adhere to and invade kidney epithelial cells in culture. Braz J Med Biol Res 45: 417-424.

19. Renner LD, Weibel DB (2011) Physicochemical regulation of biofilm formation MRS Bull 36: 347-355. 
Citation: Chaudhary M, Kumar S, Payasi A (2013) Role of CSE1034 in Escherichia coli Biofilm Destruction. J Microb Biochem Technol 5: $054-058$. doi:10.4172/1948-5948.1000100

20. Bridier A, Le Coq D, Dubois-Brissonnet F, Thomas V, Aymerich S, et al. (2011) The spatial architecture of Bacillus subtilis biofilms deciphered using a surfaceassociated model and in situ imaging. PLoS One 6: e16177.

21. Lambert RJ, Hanlon GW, Denyer SP (2004) The synergistic effect of EDTA antimicrobial combinations on Pseudomonas aeruginosa. J Appl Microbiol 96: 244-253.

22. Raad II, Fang X, Keutgen XM, Jiang Y, Sherertz R, et al. (2008) The role of chelators in preventing biofilm formation and catheter-related bloodstream infections. Curr Opin Infect Dis 21: 385-392.
23. Al-Bakri AG, Othman G, Bustanji Y (2009) The assessment of the antibacterial and antifungal activities of aspirin, EDTA and aspirin-EDTA combination and their effectiveness as antibiofilm agents. J Appl Microbiol 107: 280-286.

24. Prakash B, Veeregowda BM, Krishnappa G (2003) Biofilms: A survival strategy of bacteria. Curr Sci 85: 1299-1307.

25. Jones KM (2012) Increased production of the exopolysaccharide succinoglycan enhances Sinorhizobium meliloti 1021 symbiosis with the host plant Medicago truncatula. J Bacteriol 194: 4322-4331. 